\title{
Quality of life, clinical outcomes and safety of early prophylactic levothyroxine administration in patients with Graves' hyperthyroidism undergoing radioiodine therapy: a randomized controlled study
}

\author{
David Taïeb ${ }^{1,2}$, Claire Bournaud ${ }^{3}$, Marie-Claude Eberle ${ }^{4}$, Bogdan Catargi $^{5}$, \\ Claire Schvartz ${ }^{6}$, Marie-Béatrice Cavarec7, Isabelle Faugeron', \\ Marie-Elisabeth Toubert ${ }^{9}$, Danielle Benisvy ${ }^{10}$, Cendrine Archange', \\ Olivier Mundler ${ }^{1}$, Philippe Caron ${ }^{11}$, Ahmad Esmaeel Abdullah' ${ }^{1}$ and Karine Baumstarck ${ }^{12}$ \\ 'Department of Nuclear Medicine, La Timone University Hospital, European and Center for Research in Medical \\ Imaging, Aix-Marseille University, 264 rue Saint-Pierre 13385 Marseille Cedex 5, France, ${ }^{2}$ Inserm UMR1068 Marseille \\ Cancerology Research Center, Institut Paoli-Calmettes, Marseille, France, ${ }^{3}$ Department of Endocrinology and \\ Nuclear Medicine, GHE-Hospices Civils de Lyon and Lyon 1 University, Lyon, France, ${ }^{4}$ Department of Nuclear \\ Medicine, Lapeyronie University Hospital, Montpellier, France, ${ }^{5}$ University Hospital of Bordeaux, Bordeaux, France, \\ ${ }^{6}$ Department of Nuclear Medicine, Jean Godinot Institute, Reims, France, ${ }^{7}$ Department of Nuclear Medicine, Brest \\ University Hospital, Brest, France, ${ }^{8}$ Department of Nuclear Medicine, Saint-Antoine Hospital, Paris, France, \\ ${ }^{9}$ Department of Nuclear Medicine, Saint-Louis Hospital, Paris, France, ${ }^{10}$ Department of Nuclear Medicine, University \\ of Nice, Nice, France, ${ }^{11}$ Department of Endocrinology and Metabolic Diseases, CHU Larrey, Toulouse University \\ Hospital, Toulouse, France and ${ }^{12}$ Department of Public Health, EA3279 Self-perceived Health Assessment Research \\ Unit, La Timone University, Aix-Marseille University, Marseille, France
}

\author{
Correspondence \\ should be addressed \\ to $D$ Taïeb \\ Email \\ david.taieb@ap-hm.fr
}

\begin{abstract}
Objective: While radioiodine therapy is commonly used for treating Graves' disease, a prolonged and clinical hypothyroidism may result in disabling symptoms leading to deterioration of quality of life $(\mathrm{QoL})$ of patients. Introducing levothyroxine $\left(\mathrm{LT}_{4}\right)$ treatment in the early post-therapeutic period may be an interesting approach to limit this phenomenon.

Methods: A multicenter, prospective, open-label randomized controlled trial enrolled 94 patients with Graves' hyperthyroidism randomly assigned to the experimental group $(n=46)$ (group A: early prophylactic $\mathrm{LT}_{4}$ treatment) or the control group ( $n=48$ ) (group B: standard follow-up). The primary endpoint was the 6-month QoL. The secondary endpoints were other QoL scores such as Graves' ophthalmopathy (GO) outcomes, thyroid function tests and safety. Results: The primary endpoint at 6 months was achieved: the mental composite score (MCS) of Short Form 36 (SF-36) was significantly higher in group A compared to group B $(P=0.009)$. Four other dimension scores of the SF-36 and four dimension scores of the thyroid-specific patient-reported outcome (ThyPRO) significantly differed between the two groups, indicating better QoL in group A. After adjustment for variables, the early $\mathrm{LT}_{4}$ administration strategy was found as an independent factor for only two scores of SF-36: the MCS and the general health (GH) score. There were no differences in GO, final thyroid status and changes in the anti-TSH receptor antibodies (TRAbs) levels between the two groups. No adverse cardiovascular event was reported. Conclusion: Early $\mathrm{LT}_{4}$ administration post-radioactive iodine (RAI) could represent a safe potential benefit for patients with regard to QoL. The optimal strategy taking into account administered RAl activities and $\mathrm{LT}_{4}$ treatment dosage and timing remains to be determined.
\end{abstract} Printed in Great Britain
European Journal of Endocrinology (2016) 174, 491-502 


\section{Introduction}

Graves' disease (GD) is the commonest cause of hyperthyroidism (2\% in women and $0.4 \%$ among men) $(1,2)$. Until present, there are two approaches for treating hyperthyroid patients, conservative or radical (destructive) measures. The first is based on the use of anti-thyroid drugs (ATDs) while the second is based on the use of either iodine-131 or total thyroidectomy. In Europe, the medical option is often preferred as a first-line approach with a risk of relapse between 51 and $68 \%$ after a complete initial course of therapy (at least 12 months) $(3,4)$. Radical options are mainly indicated for recurrences, resistance to (ATDs) or after drug-related adverse events (i.e. agranulocytosis and hepatitis), but patient's preference is also a relevant criterion to select the type of treatment.

Over many decades, radioiodine therapy has been used for treating Graves' disease with excellent costeffectiveness ratios $(5,6)$ and no significant oncogenetic risks $(7,8,9,10)$. There is still no consensus regarding the most appropriate approach for treating Graves' hyperthyroidism with radioiodine $(11,12,13,14,15,16,17)$. Administered radioactive iodine (RAI) activities can be either fixed (for ablation) or adjusted for different parameters such as thyroid mass, radioiodine effective period and thyroid uptake values (for either ablation or restoration of euthyroidism) $(18,19)$. Ablative approaches are increasingly being proposed for the treatment of Graves' disease in order to decrease the risk of persistent/recurrent hyperthyroidism. When an ablative strategy is chosen, a third of patients develop hypothyroidism within the first month, two-thirds by the second month and almost all patients after 3 months of $555 \mathrm{MBq}$ activity of RAI. (20).

Therefore, it is crucial to prevent and to treat hypothyroidism after radioiodine therapy: firstly, hypothyroidism may induce deleterious symptoms such as fatigue, memory impairment, cognitive decline or depressed mood (21). These symptoms are the main cause of dissatisfaction regarding radioiodine therapy $(22,23,24)$ and may lead to deterioration of quality of life (QoL) of patients (25); secondly, the hypothyroid state may also induce indirect economic costs through lost productivity and increased absenteeism (26); thirdly, radioiodine therapy increases the risk of developing de novo Graves' ophthalmopathy (GO) or worsening a pre-existing $\mathrm{GO}(27,28,29)$, which is also associated with alteration of QoL and social isolation (30). Therefore, Tallstedt et al. (31)proposed to administer levothyroxine $\left(\mathrm{LT}_{4}\right)$ in the early post-therapeutic period ( $50 \mu \mathrm{g}$ daily started at 2 weeks post-RAI) in GD patients with minimally active GO in order to avoid profound hypothyroidism and potentially prevent de novo or worsening GO.

The aims of the present multicenter randomized controlled study were to evaluate the impact of early prophylactic (preventative) $\mathrm{LT}_{4}$ administration following radioiodine therapy for Graves' hyperthyroidism in comparison to standard follow-up on QoL, anxiety and depression, cardiovascular parameters such as blood pressure (BP) and heart rate (HR), GO outcomes, thyroid function tests and anti-thyroid-stimulating hormone (TSH) receptor antibodies levels.

\section{Subjects and methods}

\section{Design}

A multicenter, prospective, randomized, controlled, openlabel, two-parallel group study compared early prophylactic $\mathrm{LT}_{4}$ administration (group $\mathrm{A}$ ) following radioiodine therapy to standard follow-up (group B) in which patients were treated with $\mathrm{LT}_{4}$ based on TSH and/or free $\mathrm{T}_{4}\left(\mathrm{fT}_{4}\right)$ concentrations. The study protocol was designed using the recommendations of the Consolidated Standards of Reporting Trials (CONSORT) statement. Recruitment was performed in seven French nuclear medicine/endocrinology centers. The methodological support had been provided by the Clinical Research Unit, Assistance Publique-Hôpitaux de Marseille (AP-HM), France.

\section{Eligibility criteria}

The inclusion criteria were as follows: age $\geq 18$ years (without an upper limit), Graves' hyperthyroidism, absence or minimal GO (NOSPECS classification), absence of active GO (clinical activity score (CAS) $<3$ ), $\mathrm{FT}_{4}$ and/or $\mathrm{FT}_{3}$ below 1.5 times the upper reference limit before RAI, thyroid volume evaluated by US within 3 months preceding the trial $<60 \mathrm{ml}$, withdrawal of ATD 5 days prior to radioiodine therapy regardless of the type or duration of treatment with ATDs and a negative pregnancy test. All patients gave their signed informed consents for participation. GO evaluation was assessed by a specialist ophthalmologist prior to inclusion.

The exclusion criteria were as follows: presence of thyroid nodules suspicious for malignancy, injection of iodinated radiographic contrast media within the 4 weeks prior to radioiodine therapy, concurrent or previous amiodarone treatment, previous history of major 
concurrent chronic medical disorders, psychiatric disorders and chronic alcoholism.

\section{Therapeutic strategies}

For radioiodine therapy, all patients received $20 \mathrm{MBq}$ of iodine-131/gram of thyroid tissue; the thyroid volume was evaluated by thyroid US.

The two therapeutic strategies were as follows:

i) Group A: in early prophylactic $\mathrm{LT}_{4}$ treatment, patients allocated to this group received daily $\mathrm{LT}_{4}(50 \mu \mathrm{g})$ starting 15 days post-radioiodine administration. Then, the dose of $\mathrm{LT}_{4}$ was adapted to thyroid function tests at 1, 3 and 6 months.

ii) Group B: patients allocated to this group were followed up every 4 weeks and treated with $\mathrm{LT}_{4}$ when needed.

$\mathrm{LT}_{4}$ dose modification was standardized as follows:

i) TSH between 4.5 and $10 \mathrm{mU} / \mathrm{l}: \mathrm{LT}_{4} 75 \mu \mathrm{g} /$ day ( $25 \mu \mathrm{g} /$ day added in patients already treated with the preventative $50 \mu \mathrm{g} /$ day).

ii) $\mathrm{TSH}>10 \mathrm{mU} / \mathrm{l}: \mathrm{LT}_{4}$ between 1.6 and $1.8 \mu \mathrm{g} / \mathrm{kg}$ per day (while adding the dose difference in patients already treated with the preventative $50 \mu \mathrm{g} / \mathrm{day}$ ).

Patients remained off ATDs with the possibility of re-treatment during follow-up, as judged by the clinicians.

\section{Endpoints}

Primary endpoint $~$ The primary endpoint was represented by the QoL score from the mental composite score (MCS) of the Short Form 36 (SF-36), assessed at 6 months post-RAI.

\section{Secondary endpoints}

i) The QoL was further assessed by additional scores: a) the MCS of SF-36 at 1 and 12 months post-RAI; b) the physical composite score of SF-36, the 8 dimension scores of the SF-36 and the thyroid-specific patient-reported outcome (ThyPRO) dimension scores at 1, 6 and 12 months post-RAI; and c) the changes in the different QoL scores between baseline, 1, 6 and 12 months post-RAI.

ii) The level of depression and fatigue was assessed at 1 and 6 months post-RAI using the self-administered Beck Depression Inventory (BDI) and the Modified Fatigue Impact Scale (MFIS) respectively. Anxiety was assessed with the Spielberger's State-Trait Anxiety Inventory (STAI).

iii) GO: at baseline, all patients were evaluated for GO by a specialist according to the European Group on Graves' Orbitopathy (EUGOGO) recommendations (examination of severity using NOPECS classification and assessment of activity using the CAS. Following RAI, patients were screened at each study visit for GO (clinical examination, EUGOGO questionnaire) and referred to an ophthalmologist if GO appearance or aggravation was suspected or observed.

iv) Thyroid status: cure/remission was defined as either euthyroidism (without ATD) or hypothyroidism. Following RAI, hypothyroidism was defined by the presence of $\mathrm{FT}_{4}<10 \mathrm{pmol} / 1$ or $\mathrm{TSH}>4.5 \mathrm{mU} / 1$. Treatment failure was defined as the need for retreatment with anti-thyroid medication, repeat RAI or thyroidectomy after initial therapy.

v) Anti-TSH receptor antibodies levels at 3 and 12 months were compared to baseline levels in two groups.

Safety - Safety assessment was carried out at each clinical visit through the evaluation of symptoms and clinical signs of thyrotoxicosis, systolic and diastolic BPs and HR.

\section{Study outline}

Inclusion - Inclusion of patients was performed by the nuclear physicians or endocrinologists. After inclusion, patients were randomized into one of the two groups after completing the consent forms.

Randomization - Computer-generated randomized lists were drawn up before the beginning of the study by the clinical research unit of the AP-HM. The randomization was stratified by center, with a 1:1 allocation ratio.

Baseline assessment - Physical signs and symptoms were evaluated by physicians. Patients filled out the first selfadministered questionnaires including socio-demographic parameters, anxiety, depression, fatigue and QoL scales. A GO assessment was performed in addition to laboratory tests for baseline $\mathrm{TSH}, \mathrm{FT}_{4}, \mathrm{FT}_{3}$ and anti-TSH receptor antibodies

Follow-up - Follow-up visits took place at 1, 3, 6 and 12 months post-RAI. At each visit, the following data were 
recorded: physical signs, symptoms and thyroid status. Depression, fatigue and QoL were assessed using patientfilled handouts containing all the relevant questionnaires at 1, 6 and 12 months. Thyroid function tests were performed locally. A GO assessment was carried out by a specialist ophthalmologist when specific symptoms according to the EUGOGO questionnaire were present. AntiTSH receptor antibody levels were measured at 3 and 12 months post-RAI.

\section{Assessment tools}

Measurement of QoL $\triangleright$ QoL was assessed using the French versions of the SF-36 and the ThyPRO. SF-36 is a generic questionnaire (32) that has eight subscales (physical function (PF), social functioning (SF), role physical (RP), role emotional (RE), mental health $(\mathrm{MH})$, vitality (V), bodily pain (BP) and general health (GH)), with scores ranging from 0 (low) to 100 (high QoL level). Two component summary measures of SF-36, namely, PCS and MCS respectively, can be calculated. Higher scores indicate higher QoL levels. The ThyPRO is an 84-itemspecific questionnaire measuring QoL with 13 scales covering physical (four scales) and mental (two scales) symptoms, function and well-being (three scales) and participation/social function (four scales) (33). Each scale ranges between 0 and 100 with increasing scores indicating decreasing QoL (i.e. more symptoms or greater impact of disease).

Assessment of depression and anxiety $\rightarrow$ The level of depression was assessed using the self-administered BDI. The BDI score range is $0-39$, with higher scores indicating worsening depression (34).

The STAI is a self-reporting questionnaire consisting of 40 items leading to two scores, state and trait scores, ranging from 20 (absence of anxiety) to 80 (high anxiety) $(35,36)$.

The level of fatigue was assessed using the MFIS (37), describing three domains: (physical, psychological and psychosocial) with an index. Higher scores indicate higher fatigue levels.

Laboratory tests $>$ Thyroid function tests (TSH, free $\mathrm{T}_{4}$ and free $\mathrm{T}_{3}$ ) were performed with kits routinely used in each clinical center. Anti-TSH receptor antibodies were evaluated at baseline and during follow-up using one of the following two assays: Elecsys Anti-TSHRCobas (Roche Diagnostics) (functional sensitivity $0.9 \mathrm{IU} / \mathrm{l}$ ) and TRAK human DYNOtest (BRAHMS
Diagnostica GmbH, Berlin, Germany) (functional sensitivity $1.5 \mathrm{IU} / \mathrm{l})$.

Ethical aspects, laws and regulations $\triangleright$ The study had been conducted in accordance with the Helsinki declaration and the French laws and regulations (Code de la Santé Publique, article L.1121-1/Loi de Santé Publique n 2004-806 du 9 août 2004 relative à la politique de santé publique et ses décrets d'application du 27 août 2006). Regulatory monitoring was performed by the sponsor. The approval of the French authorities, including the French Ethics Committee (Comite de Protection des Personnes Sud Méditerranée II) and the French Drug and Device Regulation Agency (Agence Nationale de Sécurité du Médicament) was obtained before beginning the study. The ClinicalTrials.gov identifier is NCT01295333.

\section{Statistical analysis}

Sample size $>$ The sample size was determined in order to obtain an $80 \%$ power to detect a ten-point difference (s.D. 15 pts) in QoL at 6 months as evaluated by the MCS of SF-36. With a significant $P$ value of 0.05 , these calculations showed that a total of 36 patients per group were needed; considering a potential $20 \%$ of patients being lost to follow-up at 6 months, a total of 90 patients were needed to be included. The final sample included 48 patients in the control group (group B) and 46 patients in the experimental group (group A); the QoL at 6 months was available for 43 and 39 patients respectively. No interim analysis was planned. Data were analyzed using SPSS version 17.0 Software.

Analysis - The intention-to-treat population (including all subjects who were randomized and were at least evaluated at baseline) was used for the analysis. The normality of the variables was estimated using frequency histograms and the Shapiro test. The baseline parameters were presented per group (A and B) and compared using the $\chi^{2}$-test or Fisher's exact test for categorical variables and Student's $t$-test for continuous variables.

$\mathrm{LT}_{4}$ and ATD treatments, thyroid and ophthalmologic status were compared between the two groups.

The Mental Composite Scores of SF-36 assessed at 1, 6 (primary endpoint) and 12 months post-RAI were compared between the two groups using the MannWhitney test. The same comparison was performed for the other SF-36 and ThyPRO scores at each evaluation period (1, 6 and 12 months). 
Changes in QoL scores between baseline and 6 months respectively were compared between the two groups, and the analysis of variation for repeated measurements was performed to compare changes over time (baseline, 1 and 6 months) between the two groups. The same analysis was performed for depression and fatigue scores (baseline, 1 and 6 months).

For each 6-month QoL score that differed between the two groups, multivariate analyses using multiple linear regressions were performed to determine variables potentially predictive of QoL levels. Variables relevant to the models were selected based on their clinical interest (gender and age) and/or a threshold $P$ value $<0.05$ during univariate analysis. The final models expressed the standardized beta coefficients. The coefficient represents the change of the standard deviation in the dependent variable (QoL) resulting from a change of one standard deviation in the different independent variables. The independent variables with the higher standardized beta coefficients are the variables with a greater relative effect on QoL.

A subgroup analysis was performed comparing patients within group A (early prophylactic) that had no interruption of $\mathrm{LT}_{4}$ during the first 3 months (A1), with the patients within group $\mathrm{B}$ (standard follow-up) that had no introduction of $\mathrm{LT}_{4}$ during the first 3 months (B1).

All the tests were two-sided. Statistical significance was defined as $P<0.05$.

\section{Results}

\section{Study population}

Ninety-four patients were included in the present study. Four patients in group A were lost to follow-up before 6 months (one at 2 months, two at 3 months and one at 4 months) and three patients in group B (all at 3 months). The primary endpoint was available for 39 and 43 patients in groups A and B, respectively.

Patients' baseline characteristics at the time of RAI therapy are shown in Table 1 . The mean age was 48.8 and 47.1 years in groups $\mathrm{A}$ and $\mathrm{B}$, respectively. Sociodemographic parameters, anxiety scores (trait and state), fatigue levels and the QoL levels did not differ between the two groups at baseline except for the PCS, BP and GH scores of SF-36 and the sex-life score of ThyPRO with a significantly better QoL for group A. The mood disorders (BDI score) were significantly more frequent within group B than A.

The two groups displayed similar characteristics, with the exception of higher plasma $\mathrm{FT}_{4}$ concentrations in group $\mathrm{B}(P=0.017)$. The average administered RAI activity was similar in the two groups, $410 \pm 168$ and $410 \pm 165 \mathrm{MBq}$, respectively. Thyroid uptake values obtained in 64 patients (39 iodine-123 and 25 technetium-99m-) and urinary iodine levels assessed in 35 cases were not statistically different between the two groups.

\section{Follow-up in both groups}

At 1-month post-RAI, 7/45 (16\%) patients in group A were no longer on $\mathrm{LT}_{4}$. Three of the seven patients developed hypothyroidism 1 month later. At 2 months, three additional patients were off $\mathrm{LT}_{4}$. The decision to stop $\mathrm{LT}_{4}$ administration in this group was left to clinicians' judgment, as this subgroup of patients experienced an increase in thyroid hormone levels. However, there were no adverse cardiovascular events reported in group A patients.

In group $\mathrm{B}, \mathrm{LT}_{4}$ administration was introduced in seven patients at 1-month post-RAI, while at 2 months, 11 additional patients were started on $\mathrm{LT}_{4}$.

Very few patients in both groups required ATDs (Table 2).

\section{QoL outcomes}

At 6 months, the MCS of SF-36 (primary endpoint) was significantly higher (better QoL) in group A compared to $\mathrm{B}(P=0.009)$. Four other dimension scores of the SF-36 (RE, MH, SF and GH) and four dimension scores of the ThyPRO (emotional susceptibility, social impairment, sex life and cosmetics complaints) significantly differed between the two groups, indicating better QoL in group A. QoL changes between baseline and 6 months did not differ between the two groups except in certain domains only (data not shown). A statistically significant difference in the variation over time (baseline, 1 and 6 months) of QoL scores was observed between the groups for: i) SF-36: MCS (Figure 1) and five scores (RE, MH, SF, BP and GH) and ii) ThyPRO: the cosmetics complaints score; group B showed lower levels than A $(P<0.05)$.

A subgroup analysis was performed after excluding patients that were retreated before 6 months or lost in follow-up. This subgroup analysis, comparing the 6-month QoL scores between the 32 individuals of group A (A1: early prophylactic without interruption of $\mathrm{LT}_{4}$ during the first 3 months) and the 34 individuals of group B (B1: standard follow-up without introduction of $\mathrm{LT}_{4}$ during the first 3 months), showed significantly higher QoL scores in most domains of SF-36 (RE, RP, MH, SF, GH and MCS) and ThyPRO 
Table 1 Baseline characteristics.

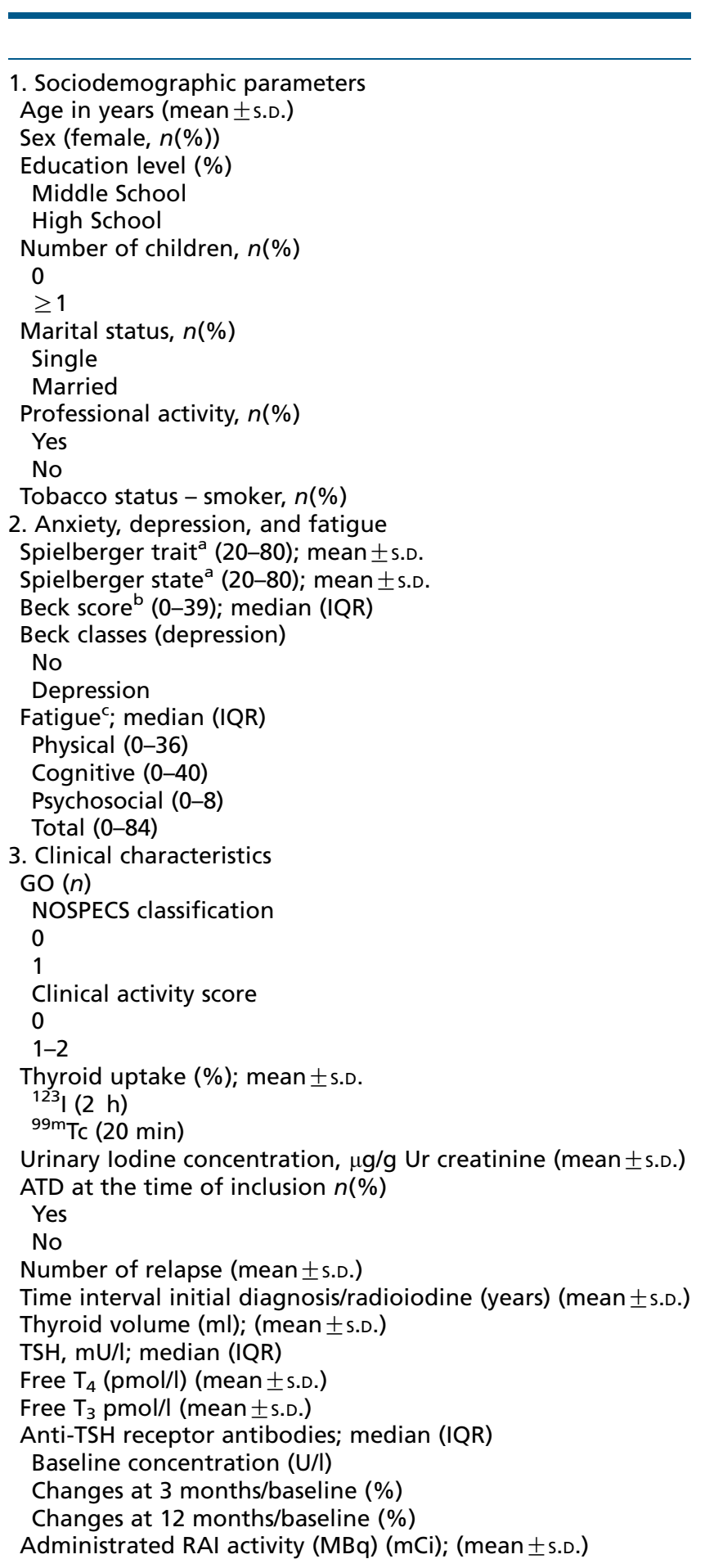

3. Clinical characteristics

NOSPECS classification

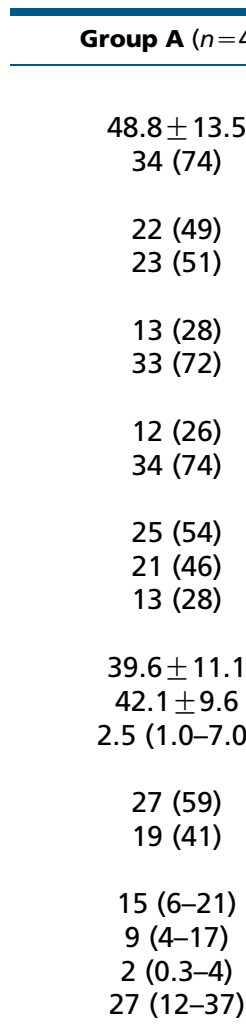

P.

\begin{tabular}{|c|c|}
\hline Group B $(n=48)$ & $\boldsymbol{P}$ \\
\hline $\begin{array}{c}47.1 \pm 12.9 \\
37(77)\end{array}$ & $\begin{array}{l}0.544 \\
0.721 \\
0.268\end{array}$ \\
\hline $\begin{array}{l}18(38) \\
30(63)\end{array}$ & \\
\hline $\begin{array}{c}7(15) \\
41(85)\end{array}$ & 0.105 \\
\hline $\begin{array}{l}14(29) \\
34(71)\end{array}$ & 0.739 \\
\hline $\begin{array}{l}27(56) \\
21(44)\end{array}$ & 0.853 \\
\hline $17(35)$ & 0.457 \\
\hline $\begin{array}{c}41.1 \pm 11.8 \\
43.3 \pm 9.4 \\
4.5(2.3-10.0)\end{array}$ & $\begin{array}{l}0.536 \\
0.530 \\
0.037 \\
\mathbf{0 . 0 1 4}\end{array}$ \\
\hline $\begin{array}{l}16(33) \\
32(67)\end{array}$ & \\
\hline $\begin{array}{c}17(8-26) \\
15(5.3-23) \\
4(0.8-5.3) \\
32(17-53)\end{array}$ & $\begin{array}{l}0.266 \\
0.136 \\
0.125 \\
0.129\end{array}$ \\
\hline
\end{tabular}

44

2

46

2

$$
23 \pm 16
$$$$
7 \pm 5
$$$$
180 \pm 151
$$

$$
24 \text { (53) }
$$

$21(47)$

$2.4 \pm 1.0$

$6.46 \pm 5.57$

$20.4 \pm 10.4$

$0.19(0.01-1.47)$

$15.6 \pm 5.1$

$5.9 \pm 2.6$

$4.18(1.79-7.98)$

$+296.0(13-540)$

+39.5 (-17-106)

$410 \pm 168$

$(11 \pm 4.5)$

\subsection{7}

0.495

48

0

$$
\begin{gathered}
28 \pm 26 \\
7 \pm 7 \\
161 \pm 122
\end{gathered}
$$

23 (49)

24 (51)

$2.0 \pm 0.7$

$5.2 \pm 4.4$

$21.5 \pm 10.3$

0.07 (0.01-1.29)

$19.0 \pm 8.3$

$7.5 \pm 4.6$

$3.70(1.63-5.90)$

$+373.0(0-830)$

+48.0 (-29-539)

$410 \pm 165$

$(11 \pm 4.5)$
0.388

0.887

0.691

0.673

0.102

0.240

0.595

0.404

0.017

0.068

0.314

0.825

0.325

0.995

According to the eligibility criteria, ATDs were withdrawn 5 days prior to radioiodine therapy

${ }^{a}$ Higher score, higher anxiety level.

bHigher score, more severe depression.

'Higher score, higher fatigue level. 
Table 2 Evolution of $\mathrm{LT}_{4}$ and ATD usage and percentages of change in $\mathrm{FT}_{4}$ during the first 3 months post-RAI.

\begin{tabular}{l}
\hline \\
\hline Number of patients on- $\mathrm{LT}_{4}$ at: \\
1 month \\
2 months \\
3 months \\
Number of patients on ATD at: \\
1 month \\
2 months \\
3 months \\
$\%$ variation $\mathrm{FT}_{4}:$ \\
1 month/baseline \\
Mean \pm S.D. \\
Median (IQR) \\
2 months/baseline \\
Mean \pm S.D. \\
Median (IQR) \\
3 months/baseline \\
Mean \pm S.D. \\
Median (IQR)
\end{tabular}

\begin{tabular}{ccc}
\hline Group B $(n=48)$ & & $\boldsymbol{P}$ \\
$7 / 48$ & $<10^{-3}$ \\
$18 / 48$ & $<10^{-3}$ \\
0 & $<10^{-3}$ \\
2 & $\mathrm{NS}$ \\
4 & $\mathrm{NS}$ \\
& $\mathrm{NS}$ \\
$-2.9 \pm 47.3$ & $<10^{-3}$ \\
$-9.3(-30.2 ; 17.5)$ & \\
$-3.9 \pm 75.2$ & 0.114 \\
$-16.0(-55.4 ; 32.2)$ & \\
$-18.2 \pm 43.4$ & 0.045 \\
$-23.5(-46.9 ; 13.8)$ & \\
\hline
\end{tabular}

NS, non-significant.

(anxiety, social impairment, daily life, sex life and cosmetics complaints). These findings are illustrated in Fig. 2.

Final thyroid status and ophthalmologic outcomes • The thyroid status of patients is detailed in Table 3 .

The majority of patients achieved euthyroidism (without ATDs) or hypothyroidism at 12 months postRAI; 92.8\% (83.3\% hypothyroidism and 9.5\% euthyroidism) and 93.3\% (77.8\% hypothyroidism and 15.5\% euthyroidism) in group A and B respectively (Table 3). The levels of anti-TSH receptor antibodies were similar at baseline as were the changes during the 1-year follow-up after RAI treatment (Table 1).

Only two patients (one in each group) developed GO: (2.4 and $2.2 \%$ in group A and B respectively).

\section{Safety}

There were no adverse cardiovascular events reported throughout the study with no statistical difference between $\mathrm{BP}$ and $\mathrm{HR}$ readings between groups $\mathrm{A}$ and $\mathrm{B}$. The median BP at 6 months was $126.5 / 75$ and 130/80 mmHg for group A and B respectively.

In subgroup analysis, no single variable showed any statistically significant difference at any point of time.

\section{Depression and fatigue}

As depression was unbalanced at baseline between groups, the 1 and 6 months scores were not different. The fatigue levels and the variations over time (baseline, 1 and 6 months) did not differ between the two groups (data not shown).

\section{Predictors of health quality outcomes}

In order to determine the real effect of early $\mathrm{LT}_{4}$ treatment on the 6-month QoL scores, multivariate models were performed with adjustment of the following variables: age, gender and unbalanced baseline parameters $(P<0.05)$, i.e. the depression score and the plasma free $\mathrm{T}_{4}$ levels. An independent model was performed for each 6-month QoL

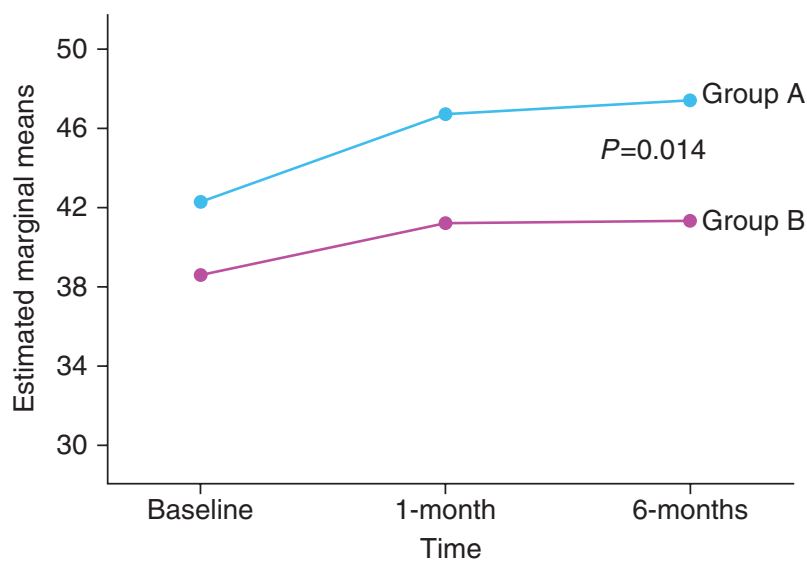

\section{Figure 1}

Variation overtime of the MCS of SF-36 in both groups, with statistical significance at 6 months. 


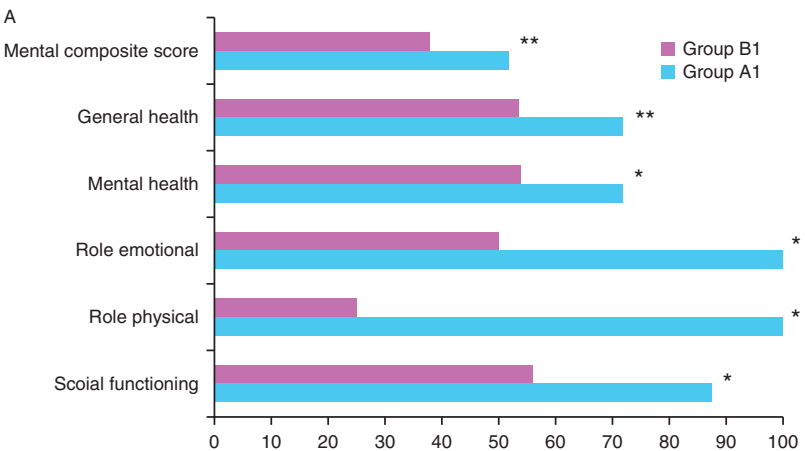

B

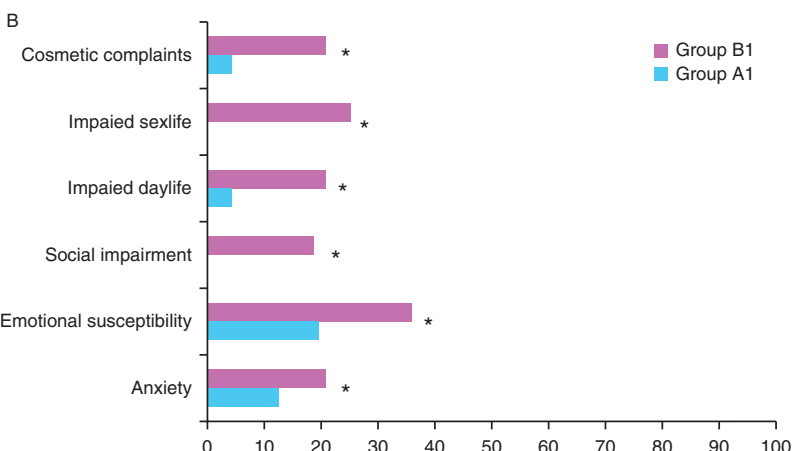

Figure 2

Comparison in QoL scores between early prophylactic without interruption of $\mathrm{LT}_{4}(\mathrm{~A} 1)$ vs standard follow-up without introduction of $\mathrm{LT}_{4}$ (B1). A: SF-36; B: ThyPRO; *: $P<0.05$ **: $P<0.01$.

score that differed between the two groups: MCS, RE, MH, SF and GH for SF-36, and ES, SI, SL and CC for ThyPRO (Table 4). After adjustment, the early $\mathrm{LT}_{4}$ treatment strategy was found as an independent factor for only two scores of SF-36, the MCS and the GH score. Depression was also associated with all the 6-month QoL scores. All the details are presented in Table 5.

\section{Discussion}

To the best of our knowledge, this is the first randomized controlled study that evaluates the impact of early $\mathrm{LT}_{4}$ administration post-RAI on QoL in patients with Graves' disease. Furthermore, homogeneity was achieved by standardizing the administered radioiodine activities based on thyroid mass, along with standardized $\mathrm{LT}_{4}$ administration regimens throughout the entire study period.

The principal conclusions that can be drawn from this study include: i) a slightly better QoL associated with early $\mathrm{LT}_{4}$ administration post-RAI therapy, which is not associated with adverse cardiovascular events or significant increases in BP or HR; ii) a high success rate of thyroid volume-adjusted RAI in both groups; and iii) a lower rate of radioiodine-induced ophthalmopathy (RAI-induced GO) in the study population compared to previous studies despite the short-term follow-up.

At 6 months post-RAI, the QoL of patients with early systematic $\mathrm{LT}_{4}$ administration appeared significantly better than that of patients allocated to the standard protocol. Although this benefit in QoL scores appeared modest, this improvement was observed in several domains of the generic and the specific QoL questionnaires. While the objective control of the disease did not really differ between the two strategies, it is now wellrecognized that the parameters of disease control and the physicians' perceptions do not reflect all the aspects that patients consider important in their lives (38). Patient-reported outcomes (PROs) can provide supplementary clinical relevance in the evaluation of disease progression, treatment and the management of care provided to patients with chronic disease. Large international agencies encourage and recommend the assessment of QoL and psychological well-being $(39,40)$ and the CONSORT statement provides recent evidence-based recommendations to improve the completeness of reporting of PROs (41).

In terms of subgroup analysis, the difference in QoL scores was well-observed between group A patients who remained on $\mathrm{LT}_{4}$ (subgroup A1) vs group B patients who had not yet had $\mathrm{LT}_{4}$ introduced during the first 3 months (subgroup B1).

As observed from Table 4, there were no significant differences in QoL scores between group A and B at 1 and 12 months respectively. This could be explained by the fact that differences in thyroid status were less evident at those periods.

Unfortunately, depression levels at baseline were not similar between the two groups. However, after

Table 3 Clinical outcomes after RAI.

\begin{tabular}{|c|c|c|c|}
\hline & Group A $(n=46)$ & Group B $(n=48)$ & $P$ value \\
\hline Hypothyroidism ${ }^{a}$ & $35 / 42(83.3 \%)$ & $35 / 45(77.8 \%)$ & \\
\hline Euthyroidism $^{a}$ & $4 / 42(9.5 \%)$ & $7 / 45(15.5 \%)$ & $0.723^{b}$ \\
\hline Treatment failure & $3 / 42(7.1 \%)^{c}$ & $3 / 45(6.7 \%)^{d}$ & \\
\hline De novo GO & $1 / 42(2.4 \%)$ & $1 / 45(2.2 \%)$ & 1.000 \\
\hline $\begin{array}{l}\text { Lost to follow-up } \\
<12 \text { months }\end{array}$ & 4 & 3 & 0.711 \\
\hline
\end{tabular}

${ }^{\text {a } T h y r o i d ~ s t a t u s ~ a t ~} 12$ months post-RAl.

${ }^{b}$ Freeman-Halton test.

${ }^{\mathrm{C}} \mathrm{All}$ three patients re-treated with $\mathrm{RAl}$ at 6,8 and 12 months respectively. ${ }^{\mathrm{d}}$ One was operated at 4 months while the remaining two received ATD. 


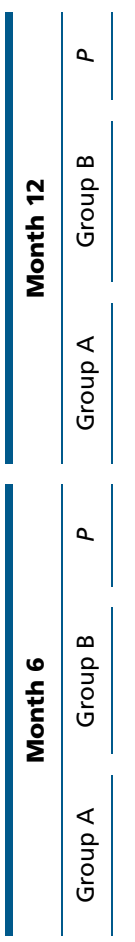

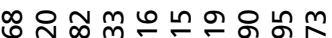

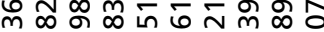
0000000000

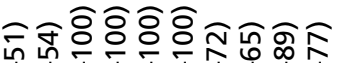

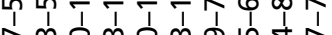

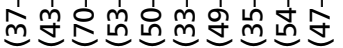

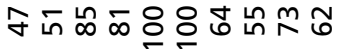

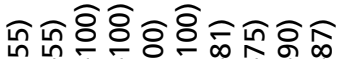

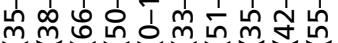

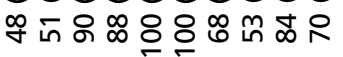

- $\quad$ ㅇํำ

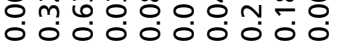

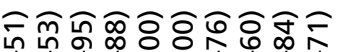

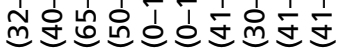

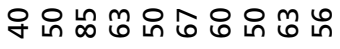

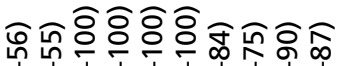

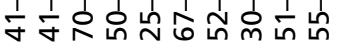

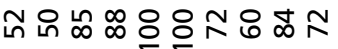

a

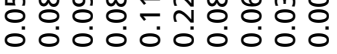

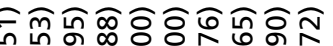

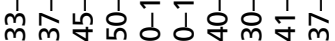
ব得

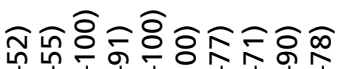

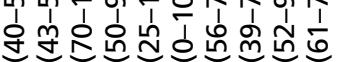

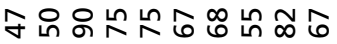

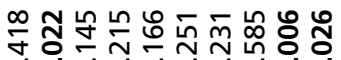

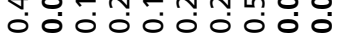

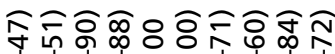

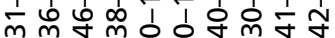

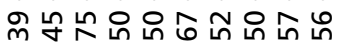

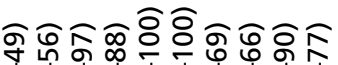

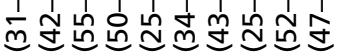

于守织

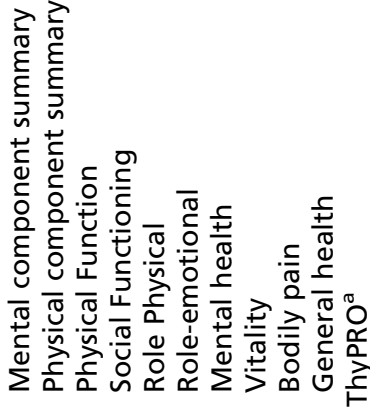

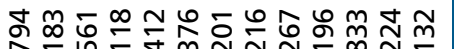

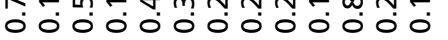

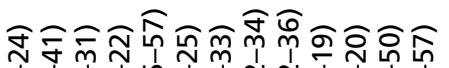

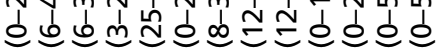
๑유읭ำ

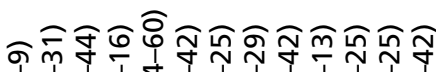

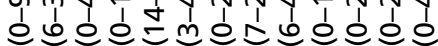
oma m

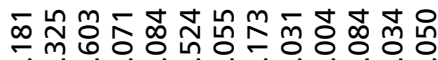
०000000 00.000.

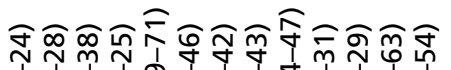
ó

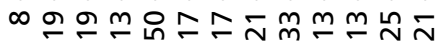

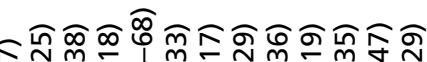

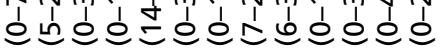

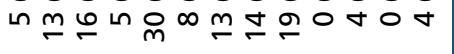

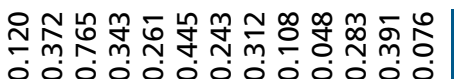

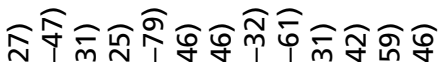
İ

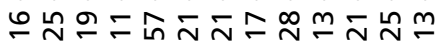

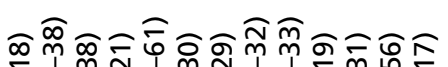

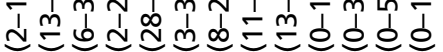

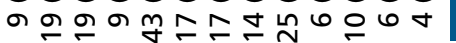

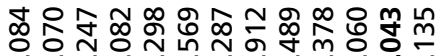

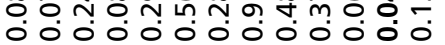

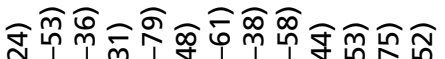

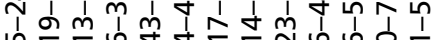
이의

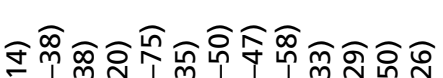

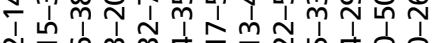

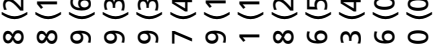

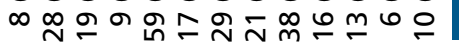


Table 5 Impact of early $\mathrm{LT}_{4}$ administration on QoL dimensions at 6 months after adjustment (multivariate analysis, standardized beta coefficients).

\begin{tabular}{|c|c|c|c|c|c|c|c|c|c|c|}
\hline & & \multicolumn{5}{|c|}{ SF-36 } & \multicolumn{4}{|c|}{ ThyPRO } \\
\hline & & MCS & $\mathrm{RE}$ & $\mathrm{MH}$ & SF & $\mathrm{GH}$ & ES & SI & SL & $\mathrm{CC}$ \\
\hline \multirow[t]{2}{*}{ Age } & $\beta$ & -0.172 & -0.295 & -0.150 & -0.129 & -0.013 & 0.082 & 0.096 & 0.078 & -0.108 \\
\hline & $P$ & 0.08 & 0.003 & 0.122 & 0.194 & 0.901 & 0.411 & 0.333 & 0.909 & 0.251 \\
\hline \multirow[t]{2}{*}{ Gender $^{\#}$} & $\beta$ & -0.082 & 0.012 & -0.174 & -0.126 & -0.117 & 0.065 & 0.107 & -0.038 & 0.149 \\
\hline & $P$ & 0.413 & 0.905 & 0.082 & 0.216 & 0.262 & 0.531 & 0.296 & 0.736 & 0.128 \\
\hline \multirow[t]{2}{*}{ Baseline $\mathrm{FT}_{4}$} & $\beta$ & 0.043 & -0.004 & 0.017 & 0.034 & 0.105 & -0.020 & 0.023 & -0.025 & 0.054 \\
\hline & $P$ & 0.661 & 0.965 & 0.858 & 0.735 & 0.306 & 0.839 & 0.816 & 0.825 & 0.574 \\
\hline \multirow[t]{2}{*}{ BDI } & $\beta$ & -0.421 & -0.420 & -0.415 & -0.410 & -0.324 & 0.473 & 0.471 & 0.392 & 0.507 \\
\hline & $P$ & $<10^{-3}$ & $<10^{-3}$ & $<10^{-3}$ & $<10^{-3}$ & 0.003 & $<10^{-3}$ & $<10^{-3}$ & 0.001 & $<10^{-3}$ \\
\hline \multirow[t]{2}{*}{ Group $^{\circ}$} & $\beta$ & -0.207 & -0.172 & -0.158 & -0.162 & -0.286 & 0.093 & 0.042 & 0.114 & 0.118 \\
\hline & $P$ & 0.042 & 0.086 & 0.114 & 0.113 & 0.007 & 0.365 & 0.685 & 0.325 & 0.227 \\
\hline
\end{tabular}

B, standardized beta coefficient ( $\beta$ represents the change of the s.D. in QoL score resulting from a change of one s.D. in the independent variable). 0 man, 1 woman; ${ }^{\circ} 0$ group A, 1 group $B$; Bold values: $P<0.05$.

adjustment, only two dimensions of the generic QoL questionnaire were impacted by the early $\mathrm{LT}_{4}$ administration strategy. Meanwhile, all the dimensions were influenced by the depressed mood that appeared as a strong predictor of lower QoL (42). Surprisingly, in our study, the generic questionnaires outperformed the thyroid-specific questionnaire in detecting differences over time. In comparison with the open-label design of the study, a double-blind study with the use of a placebo in the control group would have provided a higher level of evidence (43). However, our main focus was to assess the interest of the therapeutic strategy in its globality including the inconvenience of medication intake, compared to the control strategy. We cannot, however, exclude that the open-label design of our study could have probably overestimated the QoL differences between the two groups. Moreover, since our patients had a mean diagnosis of Graves' disease of 5-6 years duration, we do not know if our results would be observed in patients treated shortly with ATD before radioactive treatment.

Radioactive treatment should be performed according to the ALARA ('as low as reasonably achievable') principle. This means that for a predefined therapeutic goal, the lower the administered activity, the better it is from the radiation safety point of view. We used a mass-adjusted calculation of radioiodine activity based on $20 \mathrm{MBq} / \mathrm{g}(0.5 \mathrm{mCi} / \mathrm{g})$ of thyroid tissue. Our results are comparable with other studies that have used more complex calculations to determine radioiodine activities. In the majority of our patients, hypothyroidism was observed, a finding that is in coherence with the administered radio ablative activities. As expected, the percentage of variation in $\mathrm{FT}_{4}$ pre-RAI and post-RAI was significantly less in group A. However, ten patients in group A had stopped $\mathrm{LT}_{4}$ by 3 months due to persistent hyperthyroidism or hyperthyroxinemia, which was even aggravated in a few patients. One could argue the use of $T_{3}$ for thyroid supplementation in order to better monitor the decline of thyroid function after radioiodine. However, this treatment is less convenient than $\mathrm{LT}_{4}$, since it has to be taken twice or three times daily.

Despite the fact that none of our patients experienced adverse cardiovascular events including those with persistent hyperthyroidism, we have to consider the relatively young age of the population included in our trial: mean age of 48.8 and 47.1 years in groups A and B respectively, who had only moderate thyrotoxicosis prior to inclusion. It is worth noting that this could have had more serious outcomes in fragile or at risk populations with more severe hyperthyroidism prior to RAI. Additionally, one must be careful before extrapolating these findings to elderly patients that often have higher cardiovascular morbidity and who may require transient treatment with ATDs after RAI.

As shown from our results, the rate of RAI-induced GO was about $2 \%(2 / 94)$ which is lower compared to previous studies. In fact, different rates of RAI-induced GO have been reported in the literature largely due to heterogeneity of patient populations. The main recognized predictors of RAI-induced GO are: smoking, highly elevated pretreatment titers of anti-TSH receptor antibodies, significant hyperthyroidism and severe and prolonged post-RAI hypothyroidism with either suboptimal or delayed $\mathrm{LT}_{4}$ supplementation. In addition, an exaggerated post-RAI increase of anti-TSH receptor antibodies could also be considered as a risk factor for RAI-induced $\mathrm{GO}^{49}$. Different approaches have been explored to minimize the risk of 
RAI-induced GO, for example a better selection of candidates for RAI, improved management of iatrogenic hypothyroidism and the prophylactic use of glucocorticoids. Interestingly, the lower rate of RAI-induced GO in our study could be related to the inclusion criteria of the protocol such as exclusion of preexisting active GO, relatively small thyroid glands, well-controlled hyperthyroidism prior to treatment, regular follow-up post-RAI, long duration of Graves' disease with low levels of antiTSH receptor antibodies, in addition to a low percentage of active smokers among our patients.

\section{Conclusion}

Early administration of $\mathrm{LT}_{4}$ post-RAI seems to represent a safe potential benefit for patients with regard to QoL. This could be even more beneficial when higher activities of radioiodine are used, since the risk of severe and early hypothyroidism is greater. The optimal strategy taking into account administered RAI activities and $\mathrm{LT}_{4}$ administered dosages and timing remains to be determined by more randomized controlled clinical studies.

\section{Declaration of interest}

The authors declare that there is no conflict of interest that could be perceived as prejudicing the impartiality of the research reported.

\section{Funding}

The sponsor of the study was the Assistance Publique-Hôpitaux de Marseille (AP-HM, France). This work was supported by institutional grants from the French 2010 Regional Program of Clinical Research (Programme Hospitalier de Recherche Clinique Regional). The French version of ThyPRO and its scoring instructions were kindly provided by Dr Torquill Watt from the department of Endocrinology, Copenhagen University Hospital Rigshospitalet, Copenhagen, Denmark.

\section{References}

1 Brent GA. Clinical practice. Graves' disease. New England Journal of Medicine 2008358 2594-2605. (doi:10.1056/NEJMcp0801880)

2 Bartalena L. Diagnosis and management of Graves disease: a global overview. Nature Reviews. Endocrinology 20139 724-734. (doi:10.1038/ nrendo.2013.193)

3 Abraham P, Avenell A, Park CM, Watson WA \& Bevan JS. A systematic review of drug therapy for Graves' hyperthyroidism. European Journal of Endocrinology 2005153 489-498. (doi:10.1530/eje.1.01993)

4 Bartalena L, Burch HB, Burman KD \& Kahaly GJ. A 2013 European survey of clinical practice patterns in the management of Graves' disease. Clinical Endocrinology 201484 115-120. (doi:10.1111/ cen.12688)

5 Ljunggren JG, Torring O, Wallin G, Taube A, Tallstedt L, Hamberger B \& Lundell G. Quality of life aspects and costs in treatment of Graves' hyperthyroidism with antithyroid drugs, surgery, or radioiodine: results from a prospective, randomized study. Thyroid $1998 \mathbf{8} 653-659$. (doi:10.1089/thy.1998.8.653)

6 Patel NN, Abraham P, Buscombe J \& Vanderpump MP. The cost effectiveness of treatment modalities for thyrotoxicosis in a U.K. center. Thyroid 200616 593-598. (doi:10.1089/thy.2006.16.593)

7 Franklyn JA, Maisonneuve P, Sheppard M, Betteridge J \& Boyle P. Cancer incidence and mortality after radioiodine treatment for hyperthyroidism: a population-based cohort study. Lancet 1999353 2111-2115. (doi:10.1016/S0140-6736(98)12295-X)

8 Hall P, Berg G, Bjelkengren G, Boice JD Jr, Ericsson UB, Hallquist A, Lidberg M, Lundell G, Tennvall J, Wiklund $\mathrm{K}$ et al. Cancer mortality after iodine-131 therapy for hyperthyroidism. International Journal of Cancer 199250 886-890. (doi:10.1002/ijc.2910500611)

9 Metso S, Auvinen A, Huhtala H, Salmi J, Oksala H \& Jaatinen P. Increased cancer incidence after radioiodine treatment for hyperthyroidism. Cancer 2007109 1972-1979. (doi:10.1002/cncr.22635)

10 Ron E, Doody MM, Becker DV, Brill AB, Curtis RE, Goldman MB, Harris BS3rd, Hoffman DA, McConahey WM, Maxon HR et al. Cancer mortality following treatment for adult hyperthyroidism. Cooperative Thyrotoxicosis Therapy Follow-up Study Group. Journal of the American Medical Association 1998280 347-355. (doi:10.1001/jama.280.4.347)

11 Alexander EK \& Larsen PR. High dose of (131)I therapy for the treatment of hyperthyroidism caused by Graves' disease. Journal of Clinical Endocrinology and Metabolism 200287 1073-1077.

12 Catargi B, Leprat F, Guyot M, Valli N, Ducassou D \& Tabarin A. Optimized radioiodine therapy of Graves' disease: analysis of the delivered dose and of other possible factors affecting outcome. European Journal of Endocrinology 1994141 117-121. (doi:10.1530/eje.0.1410117)

13 Chiovato L, Fiore E, Vitti P, Rocchi R, Rago T, Dokic D, Latrofa F, Mammoli C, Lippi F, Ceccarelli C et al. Outcome of thyroid function in Graves' patients treated with radioiodine: role of thyroid-stimulating and thyrotropin-blocking antibodies and of radioiodine-induced thyroid damage. Journal of Clinical Endocrinology and Metabolism 1998 83 40-46.

14 Horacek J \& Franklyn JA. Radioiodine treatment of Graves' hyperthyroidism. Journal of Clinical Endocrinology and Metabolism $2003 \mathbf{8 8}$ 6113. (doi:10.1210/jc.2003-031172)

15 Kalinyak JE \& McDougall IR. How should the dose of iodine-131 be determined in the treatment of Graves' hyperthyroidism? Journal of Clinical Endocrinology and Metabolism 200388 975-977. (doi:10.1210/ jc.2002-021801)

16 Leslie WD, Ward L, Salamon EA, Ludwig S, Rowe RC \& Cowden EA. A randomized comparison of radioiodine doses in Graves' hyperthyroidism. Journal of Clinical Endocrinology and Metabolism $2003 \mathbf{8 8}$ 978-983. (doi:10.1210/jc.2002-020805)

17 Weetman AP. Radioiodine treatment for benign thyroid diseases. Clinical Endocrinology 200766 757-764. (doi:10.1111/j.1365-2265. 2007.02841.x)

18 Marinelli LD, Quimby EH \& Hine GJ. Dosage determination with radioactive isotopes; practical considerations in therapy and protection. American Journal of Roentgenology and Radium Therapy 1984 59 260-281.

19 Hanscheid H, Canzi C, Eschner W, Flux G, Luster M, Strigari L \& Lassmann M. EANM Dosimetry Committee series on standard operational procedures for pre-therapeutic dosimetry II. Dosimetry prior to radioiodine therapy of benign thyroid diseases. European Journal of Nuclear Medicine and Molecular Imaging 201340 1126-1134. (doi:10.1007/s00259-013-2387-x)

20 Nwatsock JF, Taieb D, Tessonnier L, Mancini J, Dong AZF \& Mundler O. Radioiodine thyroid ablation in graves' hyperthyroidism: merits and pitfalls. World Journal of Nuclear Medicine 201211 7-11. (doi:10.4103/ 1450-1147.98731)

21 Ladenson PW. Psychological wellbeing in patients. Clinical Endocrinology 200257 575-576. (doi:10.1046/j.1365-2265.2002.01682.x)

22 Kaplan MM, Sarne DH \& Schneider AB. In search of the impossible dream? Thyroid hormone replacement therapy that treats all 
symptoms in all hypothyroid patients Journal of Clinical Endocrinology and Metabolism 200388 4540-4542. (doi:10.1210/jc.2003-031436)

23 Saravanan P, Chau WF, Roberts N, Vedhara K, Greenwood R \& Dayan CM. Psychological well-being in patients on 'adequate' doses of 1-thyroxine: results of a large, controlled community-based questionnaire study. Clinical Endocrinology 200257 577-585. (doi:10.1046/j.1365-2265.2002.01654.x)

24 Walsh JP. Dissatisfaction with thyroxine therapy - could the patients be right? Current Opinion in Pharmacology 20022 717-722. (doi:10.1016/ S1471-4892(02)00209-6)

25 Jaeschke R, Guyatt G, Cook D, Harper S \& Gerstein HC. Spectrum of quality of life impairment in hypothyroidism. Quality of Life Research 19943 323-327. (doi:10.1007/BF00451724)

26 Drummond MF, Sculpher MJ, Torrance GW, O'Brien BJ, Stoddart GL. 2005 Methods for the Economic Evaluation of Health Care Programmes. pp 1-379. $3^{\text {rd }}$ Edn. Oxford: Oxford University press.

27 Tallstedt L, Lundell G, Torring O, Wallin G, Ljunggren JG, Blomgren H \& Taube A. Occurrence of ophthalmopathy after treatment for Graves' hyperthyroidism. The Thyroid Study Group. New England Journal of Medicine 1992326 1733-1738. (doi:10.1056/NEJM199206253262603)

28 Bartalena L, Marcocci C, Bogazzi F, Panicucci M, Lepri A \& Pinchera A. Use of corticosteroids to prevent progression of Graves' ophthalmopathy after radioiodine therapy for hyperthyroidism. New England Journal of Medicine 1989321 1349-1352. (doi:10.1056/ NEJM198911163212001)

29 Traisk F, Tallstedt L, Abraham-Nordling M, Andersson T, Berg G, Calissendorff J, Hallengren B, Hedner P, Lantz M, Nystrom E et al. Thyroid-associated ophthalmopathy after treatment for Graves' hyperthyroidism with antithyroid drugs or iodine-131. Journal of Clinical Endocrinology and Metabolism 200994 3700-3707. (doi:10.1210/jc.2009-0747)

30 Terwee C, Wakelkamp I, Tan S, Dekker F, Prummel MF \& Wiersinga W. Long-term effects of Graves' ophthalmopathy on health-related quality of life. European Journal of Endocrinology 2002146 751-757. (doi:10.1530/eje.0.1460751)

31 Tallstedt L, Lundell G, Blomgren H \& Bring J. Does early administration of thyroxine reduce the development of Graves' ophthalmopathy after radioiodine treatment? European Journal of Endocrinology 1994130 494-497. (doi:10.1530/eje.0.1300494)

32 Leplege A, Ecosse E, Verdier A \& Perneger TV. The French SF-36 Health Survey: translation, cultural adaptation and preliminary psychometric evaluation. Journal of Clinical Epidemiology $1998 \mathbf{5 1}$ 1013-1023. (doi:10.1016/S0895-4356(98)00093-6)
33 Watt T, Hegedus L, Groenvold M, Bjorner JB, Rasmussen AK, Bonnema SJ \& Feldt-Rasmussen U. Validity and reliability of the novel thyroid-specific quality of life questionnaire, ThyPRO. European Journal of Endocrinology 2010162 161-167. (doi:10.1530/EJE-09-0521)

34 Beck AT, Ward CH, Mendelson M, Mock J \& Erbaugh J. An inventory for measuring depression. Archives of General Psychiatry 19614 561-571. (doi:10.1001/archpsyc.1961.01710120031004)

35 Spielberger C. 1983 Manual for the State-Trait Anxiety Inventory (form Y). Palo Alto, CA: Consulting Psychologists.

36 Spielberger C, Gorsuch RL, Lushene R. 1970 Manual for the State-Trait Anxiety Inventory (Self-Evaluation Questionnaire). Palo Alto, CA: Consulting Psychologists.

37 Kos D, Kerckhofs E, Carrea I, Verza R, Ramos M \& Jansa J. Evaluation of the modified fatigue impact scale in four different European countries. Multiple Sclerosis 200511 76-80. (doi:10.1191/1352458505ms1117oa)

38 Rothwell PM, McDowell Z, Wong CK \& Dorman PJ. Doctors and patients don't agree: cross sectional study of patients' and doctors' perceptions and assessments of disability in multiple sclerosis. BMJ 1997314 1580-1583. (doi:10.1136/bmj.314.7094.1580)

39 Apolone G, De Carli G, Brunetti M \& Garattini S. Health-related quality of life (HR-QOL) and regulatory issues. An assessment of the European Agency for the Evaluation of Medicinal Products (EMEA) recommendations on the use of HR-QOL measures in drug approval. PharmacoEconomics 200119 187-195. (doi:10.2165/00019053200119020-00005)

40 Bottomley A, Jones D \& Claassens L. Patient-reported outcomes: assessment and current perspectives of the guidelines of the Food and Drug Administration and the reflection paper of the European Medicines Agency. European Journal of Cancer 200945 347-353. (doi:10.1016/j.ejca.2008.09.032)

41 Calvert M, Blazeby J, Altman DG, Revicki DA, Moher D \& Brundage MD. Reporting of patient-reported outcomes in randomized trials: the CONSORT PRO extension. Journal of the American Medical Association 2013309 814-822. (doi:10.1001/jama.2013.879)

42 Wickwar S, McBain HB, Ezra DG, Hirani SP, Rose GE \& Newman SP. Which factors are associated with quality of life in patients with Graves' orbitopathy presenting for orbital decompression surgery? Eye 201529 951-957. (doi:10.1038/eye.2015.76)

43 Guyatt GH, Oxman AD, Vist GE, Kunz R, Falck-Ytter Y, Alonso-Coello P \& Schunemann HJ. GRADE: an emerging consensus on rating quality of evidence and strength of recommendations. BMJ 2008336 924-926. (doi:10.1136/bmj.39489.470347.AD)

Received 9 November 2015

Revised version received 7 January 2016

Accepted 11 January 2016 